\title{
DRAMATIC CHANGES IN MOLECULAR CLOUD PROPERTIES ACROSS THE ARP 299 MERGER
}

\author{
S. AALTO \\ Onsala Rymdobservatorium, Onsala, Sweden \\ Caltech, Pasadena, USA \\ S.J.E. RADFORD \\ NRAO, Tucson, USA \\ AND \\ N.Z. SCOVILLE AND A.I. SARGENT \\ Caltech, Pasadena, USA
}

Arp 299 is an IR-luminous $\left(L_{\mathrm{IR}} \approx 8 \times 10^{11} L_{\odot}\right)$ merger system of two galaxies, IC 694 and NGC 3690 , at a distance of $40 \mathrm{Mpc}$. Its proximity and richness in molecular gas make it suitable for studying the effects of gravitational interactions and starburst activity on molecular cloud distributions, dynamics, and physical conditions. Previous low resolution observations $\left(10-30^{\prime \prime}\right)$ indicated that the $\mathrm{CO} /{ }^{13} \mathrm{CO} 1-0$ intensity ratio is unusually high, $>20$, suggesting an unusual population of molecular clouds (Aalto et al. 1991; Casoli et al. 1992).

Using the Caltech six-element OVRO array we have obtained high resolution maps of CO $1-0,{ }^{13} \mathrm{CO} 1-0$ and HCN $1-0$ of the inner $1^{\prime}$ of Arp 299 (Aalto et al. 1997). We have recently completed an OVRO CO 2-1 map of the same region and a ${ }^{13} \mathrm{CO} 2-1 \mathrm{map}$ in a $33^{\prime \prime}$ field centered on IC 694 . The synthesised beams are $1 . " 0 \times 0 . " 8$ for ${ }^{12} \mathrm{CO} 2-1$ (uniform weighting); $3 .{ }^{\prime \prime} 2 \times 2 .{ }^{\prime \prime} 5$ for ${ }^{13} \mathrm{CO} 2-1$ (natural weighting); $2 .{ }^{\prime \prime} 5 \times 2 .{ }^{\prime \prime} 2$ for ${ }^{12} \mathrm{CO} 1-0$ (uniform weighting); $4 .{ }^{\prime \prime} 3 \times 3 . .^{\prime \prime} 6$ for ${ }^{13} \mathrm{CO} 1-0$ (natural weighting); $5 . " 6 \times 5 .{ }^{\prime \prime} 3$ for $\mathrm{HCN}$ 1-0 (natural weighting).

\section{The $\mathrm{CO},{ }^{13} \mathrm{CO}$, and $\mathrm{HCN}$ distributions}

The CO distribution is dominated by compact structures at the centers of IC 694 and NGC 3690, and two bright CO peaks in the region where the disks of the two galaxies overlap. Extended CO emission connects the main high surface brightness regions, and emission also extends to the south east 

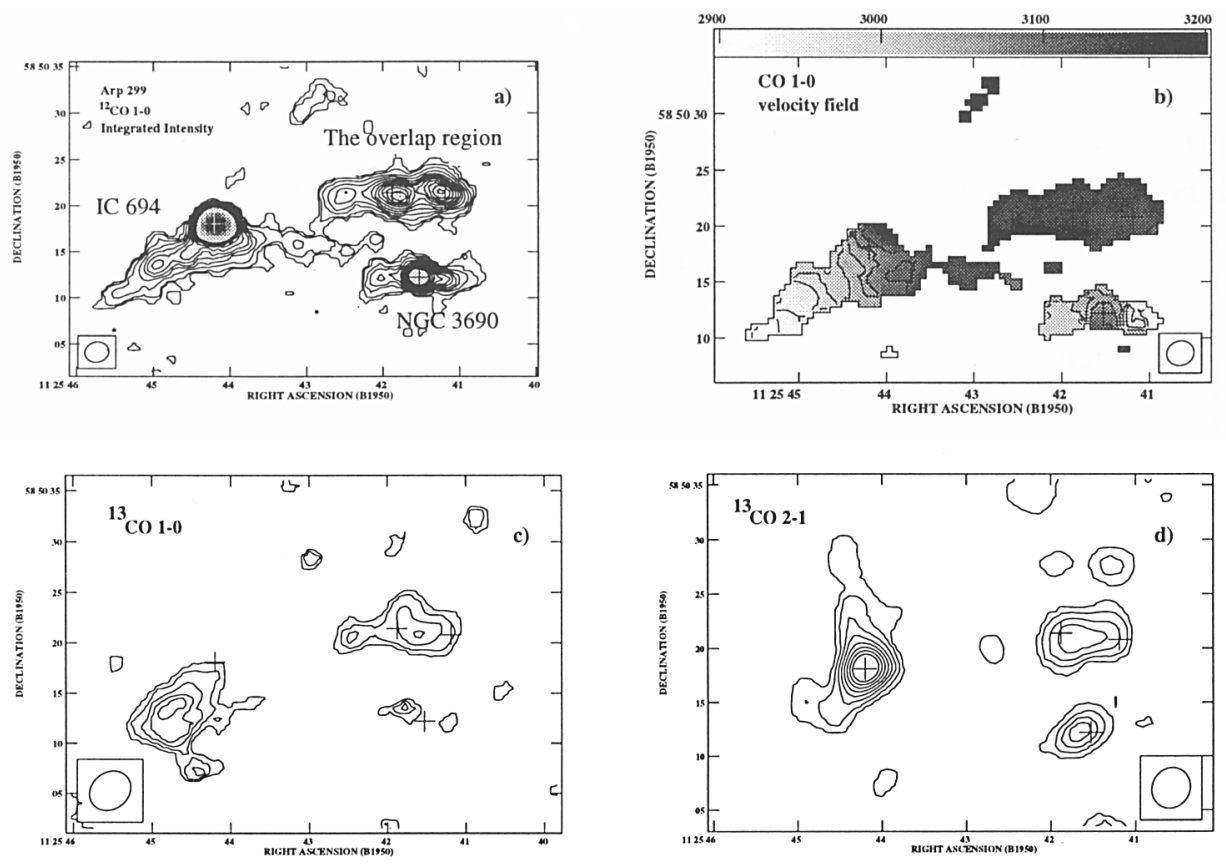

Figure 1. a) The CO 1-0 integrated intensity map (uniform weighting, contour levels are $0.9,1.8,3.6,5.4,7.2,9,10.8,12.6,14.4,16.2$, and $18 \mathrm{Jy} \mathrm{beam}^{-1} \mathrm{~km} \mathrm{~s}^{-1}$, peak flux is $97 \mathrm{Jy}_{\text {beam }}^{-1} \mathrm{~km} \mathrm{~s}^{-1}$. The crosses indicate $6 \mathrm{~cm}$ radio continuum positions (Gehrz et al. 1983). b) The CO 1-0 velocity field, the grayscale range from $2900 \mathrm{~km} \mathrm{~s}^{-1}$ to 3200 $\mathrm{km} \mathrm{s}^{-1}$. c) The ${ }^{13} \mathrm{CO} \mathrm{1-0}$ integrated intensity map (natural weighting, contour levels are $\left.0.4,0.8,1.2,1.6,2.0 \mathrm{Jy} \mathrm{beam}^{-1} \mathrm{~km} \mathrm{~s}^{-1}\right)$. d) The ${ }^{13} \mathrm{CO} \mathrm{2-1}$ integrated intensity map smoothed to the ${ }^{13} \mathrm{CO} 1-0$ resolution, (natural weighting, the lowest contour level is 1 $\mathrm{Jy} \mathrm{beam}{ }^{-1} \mathrm{~km} \mathrm{~s}^{-1}$ and the peak flux is $15 \mathrm{Jy} \mathrm{beam}^{-1} \mathrm{~km} \mathrm{~s}^{-1}$. Note NGC 3690 and the overlap region appear at the edge of the OVRO $220 \mathrm{GHz}$ primary beam and the intensities are therefore attenuated since the map has not been corrected.

of the IC 694 nucleus and to the east and west of the NGC 3690 nucleus. There is also emission north of the main features (Figure 1a).

The most striking feature of the ${ }^{13} \mathrm{CO} 1-0$ map (Figure 1c) is the $a b$ sence of strong emission at the nucleus of IC 694 and at the other strong CO peaks. Emission is detected in the disk region of IC 694, in the overlap region, and in NGC 3690 (although not at the center). Unlike Casoli et al. (1992), we find significant variation in the ${ }^{12} \mathrm{CO} /{ }^{13} \mathrm{CO} 1-0$ line ratio across Arp 299. From the very high value of $60 \pm 15$ at the IC 694 nucleus, the ratio drops to $10 \pm 3$ in the disk region, a value quite typical for normal disks of galaxies. Within the overlap region, there is an east-to-west gradient in the $\mathrm{CO} /{ }^{13} \mathrm{CO}$ intensity ratio. The highest ratio is seen in the western, most active peak. Unlike ${ }^{13} \mathrm{CO} 1-0, \mathrm{HCN} 1-0$ is brightest in the two galaxy nuclei 
and not detected in regions of extended $\mathrm{CO}$ emission. The spatial correlation between the ${ }^{13} \mathrm{CO}$ emission and radio continuum emission peaks is also poor. There is no general lack of ${ }^{13} \mathrm{CO}$ in the Arp 299 system, which one would likely expect if faint ${ }^{13} \mathrm{CO} 1-0$ emission was caused by abundance anomalies in low metallicity gas falling in from large radial distances. Instead, the extreme values of $\mathrm{CO} /{ }^{13} \mathrm{CO} 1-0$ line ratios in high $\mathrm{CO}$ surface brightness regions are mainly due to excitation effects in the gas caused by unusually high gas kinetic temperatures in regions of active star formation. The most extreme gas properties are found in the nucleus of IC 694 which may be an AGN. In the extended disk of IC 694, the cloud properties may instead be similar to those of cold giant molecular clouds in the Galaxy.

\section{Hot molecular gas in the IC 694 nucleus}

The ${ }^{13} \mathrm{CO} 2-1$ emission is dominated by the nucleus of IC 694 , instead of its disk (Fig 1d) where ${ }^{13} \mathrm{CO} 1-0$ is strongest. Hence, there are indeed ${ }^{13} \mathrm{CO}$ molecules in the inner region of IC 694, but they are highly excited. The observed ${ }^{13} \mathrm{CO} 2-1 / 1-0$ integrated intensity ratio, $3.75 \pm 0.5$, is unusually high (values close to unity are often reported for centers of starburst galaxies). The observed ${ }^{13} \mathrm{CO} 2-1 / 1-0$ ratio is close to the theoretical upper limit for optically thin, thermalized emission and sets lower limits of $100 \mathrm{~K}$ to the gas kinetic temperature and $5000 \mathrm{~cm}^{-3}$ to the gas density. It is unlikely that significant amounts of cold molecular gas prevails within the inner $3^{\prime \prime}$, since the ${ }^{13} \mathrm{CO} 2-1 / 1-0$ intensity ratio of such a gas component would be close to unity, lowering the average ratio. This means the molecular clouds of the IC 694 nucleus must be heated throughout, which may be an unusual situation. In centers of other galaxies, it is likely only the surfaces of the clouds that are heated, while their inner, denser regions remain cooler (see Aalto et al. 1994).

The ${ }^{13} \mathrm{CO} 1-0$ and 2-1 lines are optically thin. The optical depth of the CO $1-0$ line is likely to be moderate $(\tau \approx 1)$ both because the CO 2-1/10 intensity ratio is greater than unity, $\approx 1.4$ and because the $\mathrm{CO} /{ }^{13} \mathrm{CO}$ 1-0 intensity ratio is large. At a gas density of $10^{4} \mathrm{~cm}^{-3}$ and a kinetic temperature of $100 \mathrm{~K}$, the $\mathrm{CO}$ column density per velocity interval would be $\approx 10^{17} \mathrm{~cm}^{-2}\left(\mathrm{~km} \mathrm{~s}^{-1}\right)^{-1}$. The $\mathrm{CO} /{ }^{13} \mathrm{CO}$ abundance range is roughly 50 150 for the clouds in the inner region of IC 694 . This value is rather typical of clouds in the disk of the Milky Way, but higher than in the Galactic Center.

Even though intensity is attenuated at the edge of the $220 \mathrm{GHz}$ primary beam, the ${ }^{13} \mathrm{CO} 2-1$ emission is still bright in NGC 3690 and the overlap region. This further emphasizes that ${ }^{13} \mathrm{CO}$ is abundant and widespread in the Arp 299 merger. 

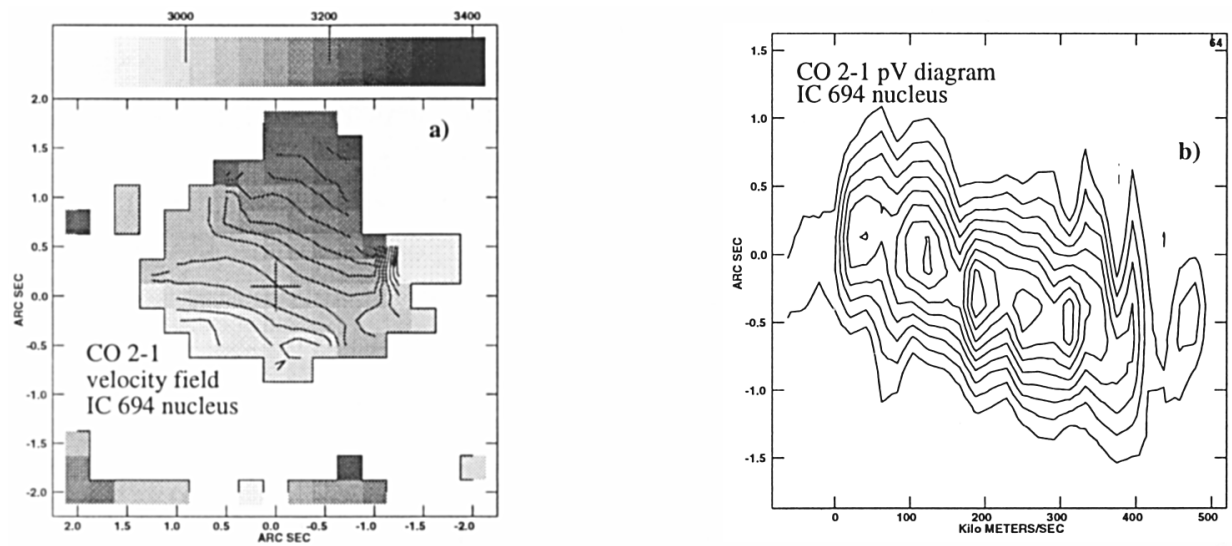

Figure 2. a) The CO 2-1 velocity field in the nucleus of IC $694\left(1 .^{\prime \prime} 0 \times 0 . .^{\prime \prime} 8\right.$ resolution). The grayscale ranges from 2950 to $3400 \mathrm{~km} \mathrm{~s}^{-1}$. b) The position velocity (pV) diagram at PA $140^{\circ}$ cut across the IC 694 nucleus.

\section{A rotating molecular disk in the center of IC 694}

The overall velocity field shows a monotonic shift from the blueshifted IC 694 disk to the redshifted overlap region and northern feature. Velocity gradients within the overlap region are small. The velocity field of NGC 3690 is complex. The structures both to the east and west of the nucleus appear blueshifted (Fig 1b).

The compact CO emission in the nucleus of IC 694 is now marginally resolved with a FWHM source size of $1 .{ }^{\prime \prime} 3 \times 0 .{ }^{\prime \prime} 8$, corresponding to a radius of $126 \mathrm{pc}$. The position angle (PA) of the central velocity field (Fig 2a) is close to $140^{\circ}$. If the central structure (Fig. $2 \mathrm{~b}$ ) rotates as a solid body, the projected velocity gradient is $200 \mathrm{~km} \mathrm{~s}^{-1}$ per $100 \mathrm{pc}$. In addition to the bright nuclear $\mathrm{CO}$ rotating at $\mathrm{PA} 140^{\circ}$ there is fainter $\mathrm{CO}$ to the east and west that participates in a different velocity pattern with a PA of $90^{\circ}$.

\section{References}

Aalto, S., Black, J.H., Johansson, L.E.B., \& Booth, R.S. 1991, A\&A, 249, 323.

Aalto et al., 1994, A\&A, 286, 365

Aalto, S., Radford, S.J.E., Scoville, N.Z., Sargent, A.I. 1997, ApJ, 475, L107

Casoli, F., Dupraz, C., Combes, F., 1992, A\&A, 264, 55

Gehrz, R.D., Sramek, R.A., \& Weedman, D.W., 1983, ApJ, 267, 551 